Biology | Cornelia Spetea

A model of photosynthesis regulation by ion fluxes in conditions of variable light

Prof Cornelia Spetea and her team at the University of Gothenburg, Sweden, study ion transport proteins and genes involved in the regulation of photosynthesis in conditions of abrupt changes in light intensity. Research on this topic is important because light fluctuations constantly occur in the natural environment and affect photosynthesis enable the Their proposed model could act as a knowledge basis to that can generate biomass for agricultural products, biofuels, and chemical feedstock in biotechnological applications.

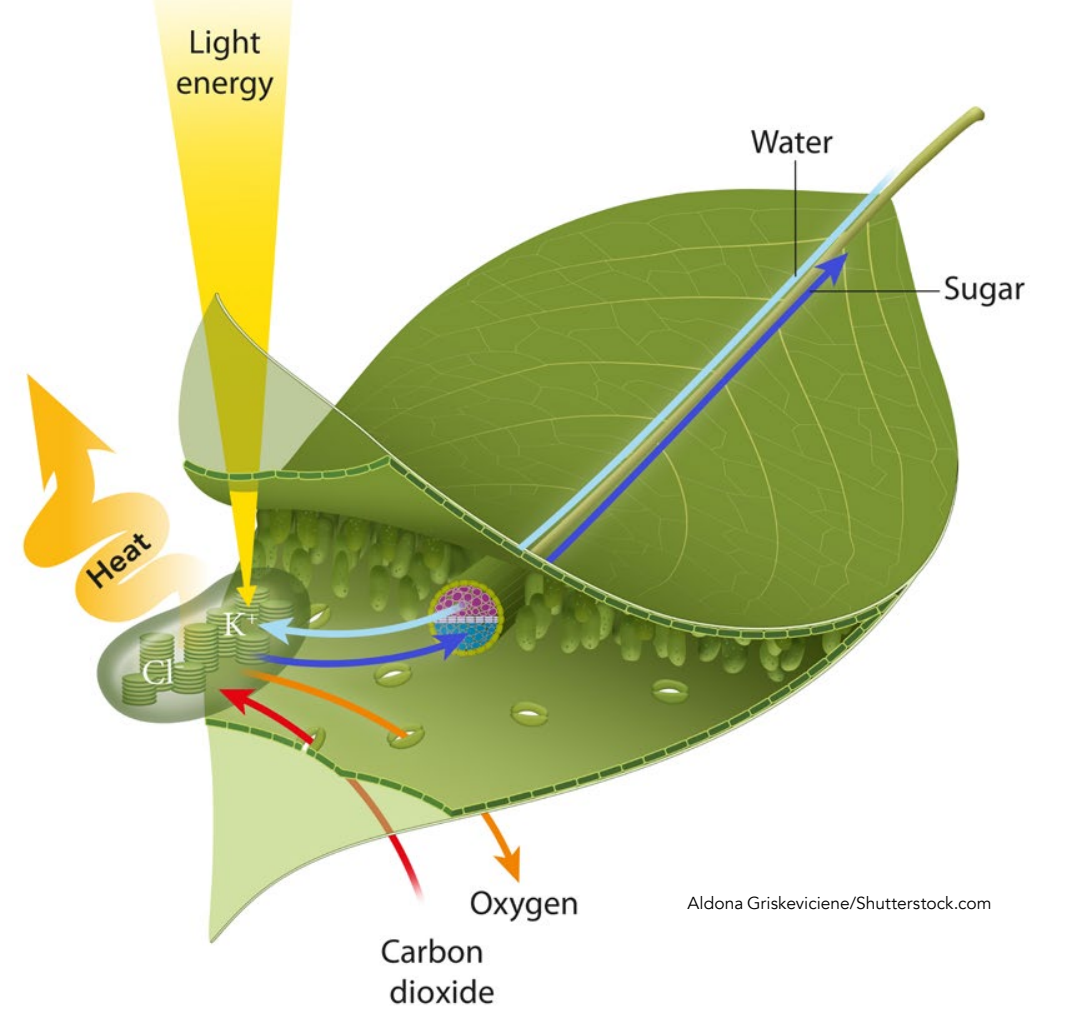

of fluctuating intensity on the model plant Arabidopsis thaliana. By following light the ref general undertanding of how to in a the energy efficiency of photosynthesis in engineered plants to make them more adapted to abrupt environment changes and to yield better crops.

\section{INVESTIGATING THE}

PHOTOPROTECTIVE RESPONSE

To identify the ion transport genes

involved in the regulation of the NPO

process in photosynthesis, Prof Spetea

and her team introduce single, double, or triple gene mutations in Arabidops thaliana. The mutations allow them to pinpoint the genes whose loss of function alters either the activation or the relaxation of the NPQ when under variable light conditions. The team published a paper in 2019
showing how changes in chlorophyll fluorescence can indicate how much of the absorbed light is used for photosynthesis and sugar production and how much is dissipated as heat in a photoprotective mechanism against excess light (NPQ). Healthy, wild-type Arabidopsis thaliana plants were able to keep a good balance of the two processes and avoided damage from excess light that could be harmfu and inhibit growth. Mutant plants, on the other hand, were affected by fluctuations in the light.

A key element of photosynthesis is the harnessing of sunlight energy via potential gradient for protons $\left(\mathrm{H}^{+}\right)$, also known as the proton motive force (PMF). Both the PMF - ultimately linked to the synthesis of ATP, the energy 'currency' of living organisms - and the photoprotective process of NPQ depend on the coordinated action of ion channels and transporters that regulate the fluxes of protons and other ions, mainly potassium $(\mathrm{K})$, chloride $\left(\mathrm{Cl}^{-}\right)$, and magnesium $\left(\mathrm{Mg}^{2+1}\right.$

UNRAVELLING THE PROTEIN NETWORK THAT FINE-TUNES PHOTOSY

and heir 2019 publcation, Prof Spetea

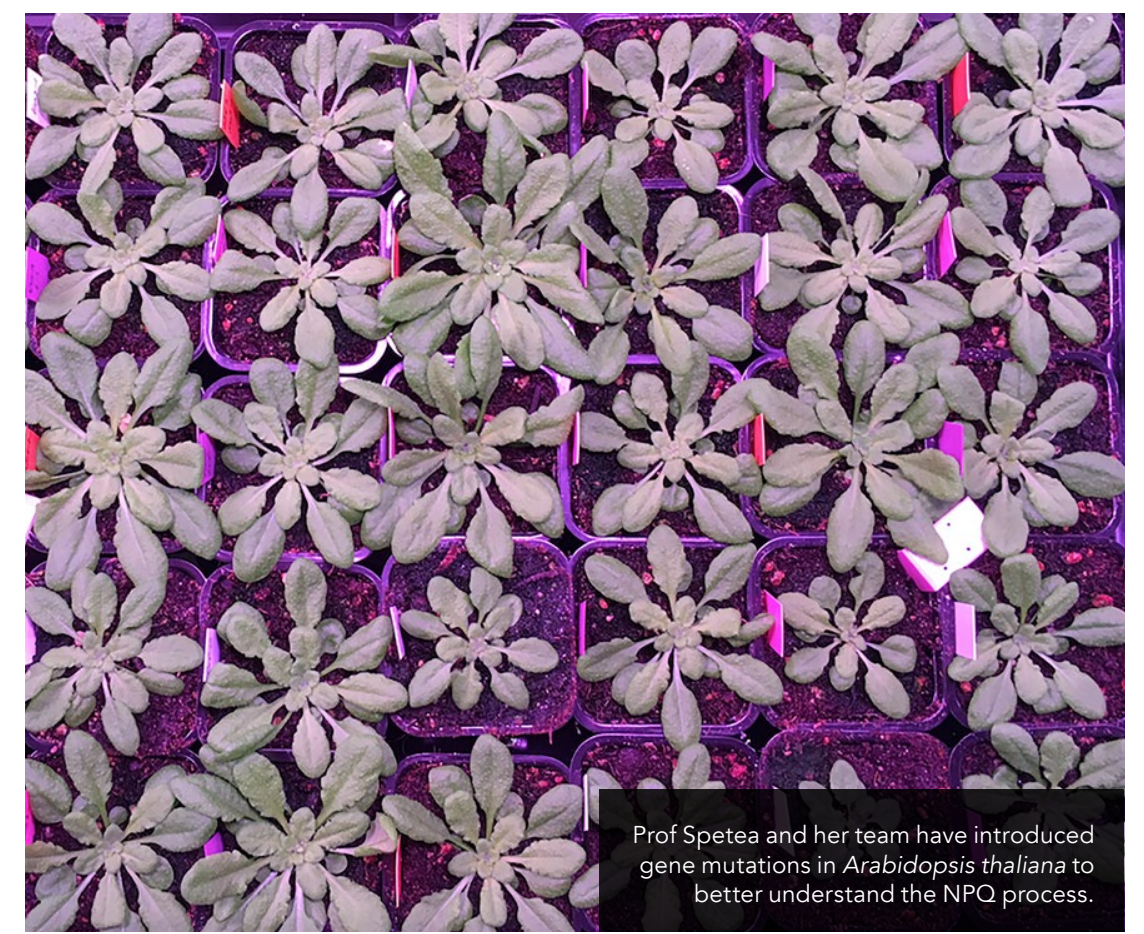

of a $\mathrm{K}^{+}$transporter, known as KEA3, and two $\mathrm{Cl}^{-}$channels, known as $\mathrm{CLCe}$ and VCCN1. These three proteins have been studied individually by the Spetea's lab; reports of their discovery and characterisation were the focus (KEA3) act publications in 2014 The data published in 2019 show that KEA3, CLCe and VCCN1 work independently of each other and that their activity is dependent on the pattern of fluctuating light intensity. KEA3 regulates photosynthesis in transition from high to low light, whereas VCCN1 acts in the transition from low two different publications in the same to high light. The effect of CLCe on

The researchers hope to achieve a general understanding of how to improve the energy efficiency of photosynthesis in engineered plants.

year). KEA3 is localised on the thylakoid photosynthesis is mainly observed in membrane, the site of light-dependent conditions of low light. By moving ions photosynthetic reactions. The between the two sides of the thylakoid transporter is responsible for the $\mathrm{pH}$ above accelerate either the activation thylakoids in exchange for $\mathrm{H}^{+}$. VCCN1 or the relaxation of NPQ, and thus fineis a voltage-dependent ion channel tune photosynthesis in variable light. responsible for the uptake of negative charges ( $\mathrm{Cl}^{-}$ions) into the thylakoids. CLCe also acts as a thylakoid-bound $\mathrm{Cl}^{-}$channel, but to date its mechanism of action has not been fully elucidated. In a previous publication (2016), Prof Spetea had proposed along with her co-authors that CLCe might play a major role in the export of $\mathrm{Cl}$ ions from upon light adaptation.

\section{ROLES OF VCCN1 AND CLCE} VCCN1 and CLCe play different roles in fluctuating light conditions. VCCN1 mediates the import of $\mathrm{Cl}^{-}$-ions into the thylakoids in a voltage-dependent manner. On transition from dark to low light, and from low to high light, phent accelerates $\mathrm{NPQ}$ activation and action of VCCN1; however, it exerts its 


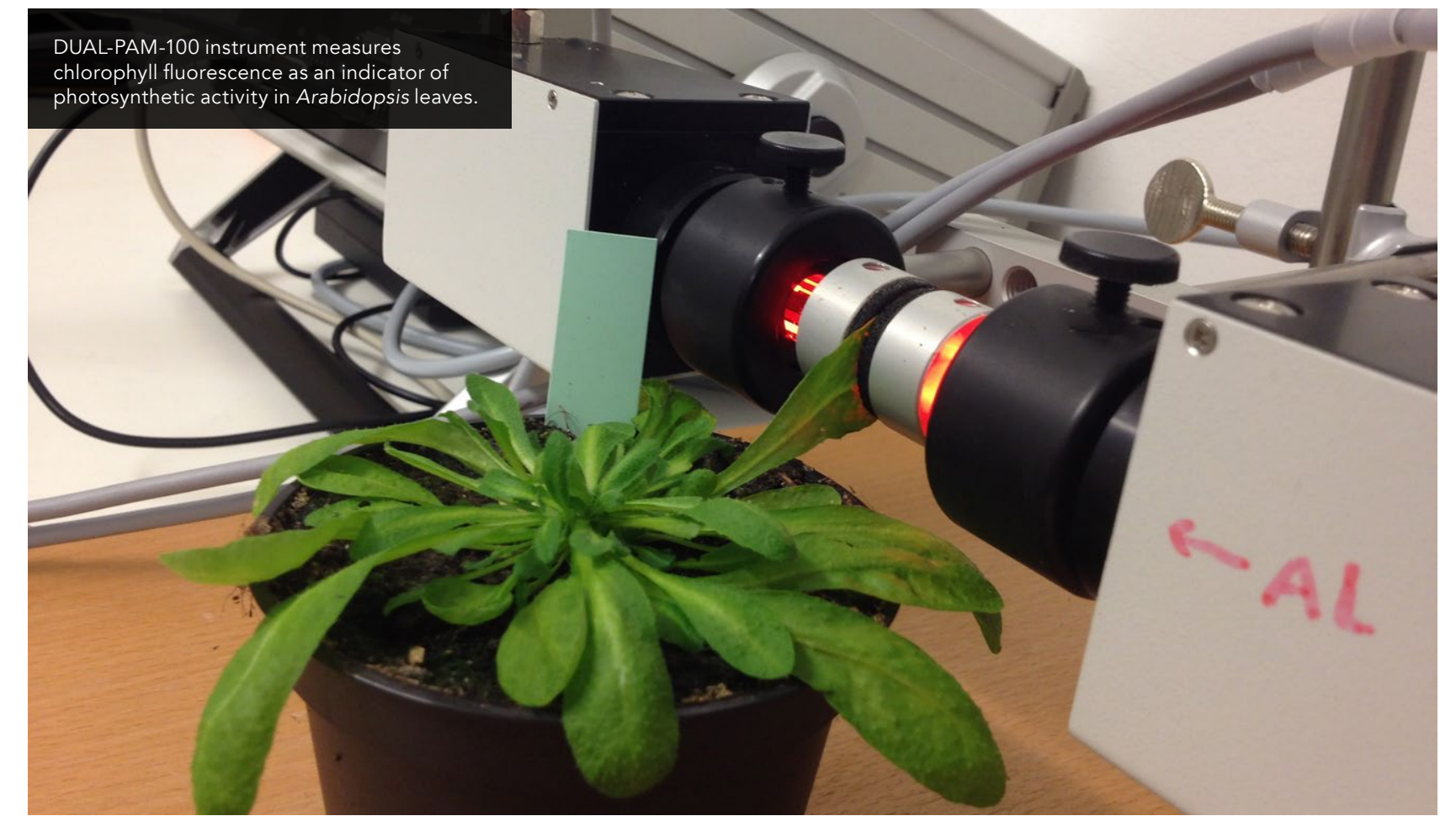

effect in low light conditions only. $\mathrm{CLCe} \quad \mathrm{VCCN} 1$ imports $\mathrm{Cl}^{-}$ions into the also upregulates the process of electron thylakoids, activating NPQ in a pHtransport in photosynthesis in a pHindependent fashion.

\section{THE ROLE OF KEA3}

KEA3 belongs to a family of six KEA

proteins and was identified as a

$\mathrm{K}^{+}$ion transporter in the thylakoid

membrane. KEA3 is inactive in high

light conditions and

is rapidly activated

upon decreasing

light intensity to

export $\mathrm{H}^{+}$out of

the thylakoids,
therefore dissipating

the $\mathrm{pH}$ gradient

- unlike VCCN1,

which builds up

If the proposed model for the NPQ process is also conserved in algae, it would provide an opportunity for large-scale production of resilient photosynthetic organisms.

photosynthesis adaptir
of fluctuating light.

\section{FUTURE DIRECTIONS} starts to increase, with the aid of $\mathrm{CLC}$. Soon after, independently of KEA3 downregulates the NPQ, State and increasing the electron transport. KEA3 Going forward, the researchers aim for other channels and transporters for other ions, such as $\mathrm{Mg}^{24}$, and lgae might have evolves ion algae might have evolved ion the similar to those observed in
Arabidopsis thaliana Arabidopsis thaliana. Algae are simpler photosynthetic organisms than plan ts
and grow faster. and grow faster. Sensitive mutants of
genes involved in photosynthesis could be grown to allow be grown to allow
for further studies accelerates the relaxation of NPQ and the chloroplast stroma, in exchange photoprotection on transition from high for $\mathrm{K}^{+}$ions, resulting in a reduced to low light. Together with $\mathrm{CLCe}$, it $\quad \mathrm{pH}$ gradient. When light intensity is reactivates electron transport.

\section{MAPPING OUT AN}

\section{OVERALL MODEL}

Taking together the data presented in 2019 and previous publications, Prof Spetea and her team propose a mode for the sequence of $\mathrm{CLCe}, \mathrm{KEA} 3$ and $\mathrm{V} C \mathrm{CN} \mathrm{N}$ are involved in the regulation of photosynthesis.
Upon transition from dark to low light, inceased from low to high, VCCN1 elevating the $\mathrm{pH}$ gradient and causing from high to low light, KEA3 exports $\mathrm{H}^{+}$out of the thylakoids decreasing $\mathrm{NPQ}$, while, in synergy with $\mathrm{CLCe}$ it reactivates the capture of light energy through the electron transport chain. The proposed model shows NPQ to increase. Upon transition

\section{and experimentation on algae. If the} proposed model for the NPQ process is also conserved in algae, it would provide an opportunity for large-scale production of resilient photosynthetic organisms that can be employed for the generation of biomass for agricultural products, fuels, and biomaterial complex area, where the pioneering advances for future generations. applications. This is a ogical an applications. This is an exciting and research of Pror Spetea and her team

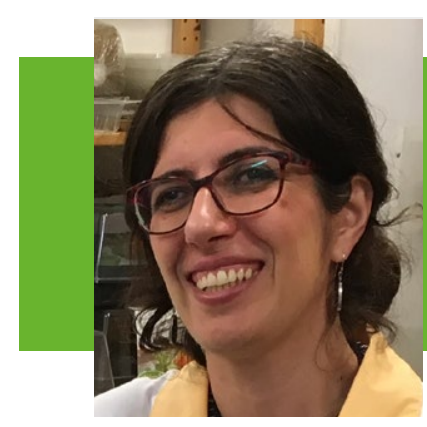

\section{Behind the Research} Prof Cornelia Spetea

E: cornelia.spetea.wiklund@bioenv.gu.se T: $+46766229332 \quad$ T: +46317869332 W: https://www.gu.se/en/research/phobio-regulation-of-photosynthesis-and-renewable biomass-production W: https://www.gu.se/en/about/find-staff/corneliaspeteawiklund
in https://www.linkedin.com/in/cornelia-spetea-wiklund-73924183/

\section{Research Objectives}

Prof Spetea studies ion transport proteins and geres involved in the regulation of photosynthesis in plants and algae.

\section{Detail}

Cornelia Spetea

Department of Biological and Environmental Sciences University of Gothenburg

Box 461

40530 Gothenburg

Sweden

Bio

Dr Cornelia Spetea has made a professional career in photosynthesis research that started with a PhD on the topic of visible and UV light photoinhibition. Her current research interests include ion transport in plants and algae and renewable biomass production from algae the west coast of Sweden.

\section{Funding}

Swesearch Council

- Carl Tryggers Foundation

\section{Collaborators}

- Andrei Herdea

- Björn Lundin

- Ildiko Szabó

- Hans-Henning Kunz

- Katalin Solymosi

- Mathias Pribil

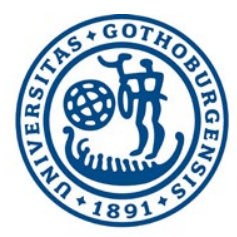

UNIVERSITY OF

GOTHENBURG

\section{References}

Dukic, E., Herdean, A., Cheregi, O., Sharma, A., Nziengui, H., Dmitruk, D., Solymosi, K., Pribil, \& M., Spetea, C. (2019). $\mathrm{K}^{+}$and $\mathrm{Cl}^{-}$- channels/transporters independently fine-tun photosynthesis in plants. Scientific reports, 9(1), 8639.
Available at: https://doi.org/10.1038/s41598-019-44972-z Kunz, H. H., Gierth, M., Herdean, A., Satoh-Cruz, M., Plastidial transporters KEA1, -2 , and -3 are essential for chloroplast osmoregulation, integrity, and $\mathrm{pH}$ regulation in Arabidopsis. Proceedings of the National Academy of Sciences of the United States of America (PNAS), 111(20), 7480-7485. Available at: https://doi.org/10.1073/ pnas. 1323899111

Herdean, A., Teardo, E., Nilsson, A. K., Pfeil, B. E., Johansson, O. N., Unnep, R., Nagy, G., Zsiros, O., Dana, S., Solymosi, K., Garab, G., Szabo, I., Spetea, C., \& Lund B. (2016). A voltage-dependent chloride channel finetunes photosynthesis in plants. Nature communications, 7, 11654. Available at: https://doi.org/10.1038/ ncomms11654

Herdean, A., Nziengui, H., Zsiros, O., Solymosi, K., Garab, G., Lundin, B., \& Spetea, C. (2016). The Arabidopsis homeostasis and regulation of photosnthens in chloride transport. Frontiers in plant science, 7 115. Available at: https://doi.org/10.3389/fpls.2016.00115

\section{Personal Response}

\section{What inspired you to conduct this research?}

II I have always been fascinated by the ability of plants to grow in and withstand various light conditions. With my knowledge in photosynthesis, I am happy I could decipher some of their secrets on how membranes and thus act in photoprotection of plants as they do it in the human eye, showing once more how much we as humans have in common with plants
at the molecular level. 